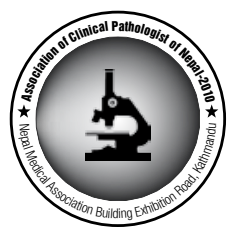

\title{
Microcystic meningioma mimicking pleomorphic xanthoastrocytoma
}

\author{
Banstola $\mathrm{S}^{1}$, Pathak $\mathrm{T}^{1}$, Neupane $\mathrm{S}^{1}$, Shrestha $\mathrm{S}^{1}$, Basyal $\mathrm{R}^{1}$, Pun $\mathrm{CB}^{1}$, Lee $\mathrm{MC}^{1}$ \\ ${ }^{1}$ Department of Pathology BP Koirala Memorial Cancer Hospital, Bharatpur , Nepal
}

\author{
Keywords: \\ MicrocysticMeningioma; \\ Pleomorphic xanthoas- \\ trocytoma Immunohisto- \\ chemistry
}

\begin{abstract}
Microcystic meningioma is a distinct morphological variant of meningioma, characterized by loose texture and microcysts with formation of large extracellular spaces containing edematous fluid. The tumor cells have stellate and vacuolated cytoplasm with long cytoplasmic processes. We report a case of microcystic meningioma occurring in a 60 year-old man. Histological features showed tumor cells with abundant foamy cytoplasm, pleomorphic nucleus with vesicular chromatin and prominent nucleoli which mimicked pleomorphic xanthoastrocytoma. The clinical presentation and histopathological diagnostic dilemma along with immunohistochemistry is discussed.
\end{abstract}

\section{INTRODUCTION}

Meningiomas constitute $13-26 \%$ of all primary central nervous system (CNS) tumors and are generally slow growing, benign tumours attached to the duramater composed of neoplastic meningothelial (arachnoidal) cells. ${ }^{1}$ They typically manifest in middle ages and elderly patients with a peak occurance during the sixth and seventh decades of life with female preponderance. Meningiomas are multiple in patients with neurofibromatosis type 2 (NF-2) and in other, non-NF-2 families with a hereditary predisposition to mengioma. Most of the meningiomas occur over the cerebral convexities, often closely associated with the falx cerebri. Most of the patients present with headache and seizure. Grossly the tumours are rubbery or firm, well circumscribed sometimes lobulated rounded masses that have a broad dural attachment. ${ }^{1}$

Cystic meningiomas are difficult to differentiate

\section{Correspondence:}

Dr Shova Banstola, MD

Registrar, Department of Pathology, BP Koirala Cancer Hospital , Bharatpur, Nepal

E-mail:nanu_sb@yahoo.com radiologically from glial tumors or metastatic tumors with a cyst. ${ }^{2}$ Correct pre-operative diagnosis is possible in only $50 \%$ of cases. ${ }^{3}$

\section{CASE REPORT}

60 years old male patient from Gulmi, Midwestern hilly area of Nepal, presented to neurosurgery outpatient department (OPD) of BP Koirala Memeorial Cancer Hospital (BPKMCH) with complaint of gradually increasing headache for 2 years which later on was associated with vertigo. There was an episode of generalized tonic clonic seizure which lasted for 2-3 minutes. On examination patient was conscious with normal higher mental functions. There was no focal neurological deficit. Magnetic resonance imaging (MRI) of head (fig.1) shows a mass occupying bifrontal region arising from falx with minimal perilesionaloedema. The patient was then subjected for surgery and the excised tumor mass was sent for histopathological evaluation. On gross examination, the mass was well circumscribed, firm in consistency measuring $6 \times 6 \mathrm{~cm}$. Cut surface showed yellowish light brown homogenous colour with cystic spaces, however haemorrhage and necrosis were absent. 

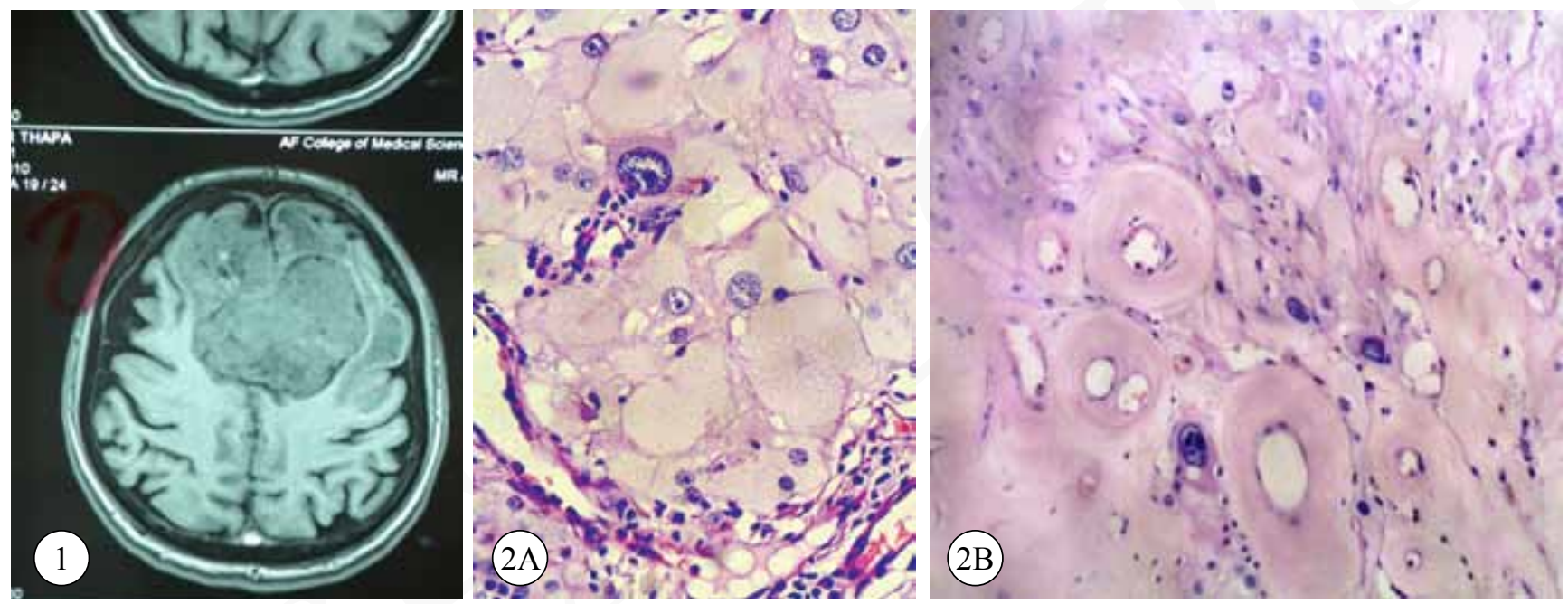

Figure 1: T1 weighted image showing hypo to isointense extraaxial mass occupying bifrontal region arising from falx.

Figure 2A: Pleomorphic tumor cells with xanthomatous change in the cytoplasm (HE stain, X40).

Figure 2B: Pleomorphic tumor cells with xanthomatous cytoplasm and hyalinized blood vessel wall (HE stain, X20).
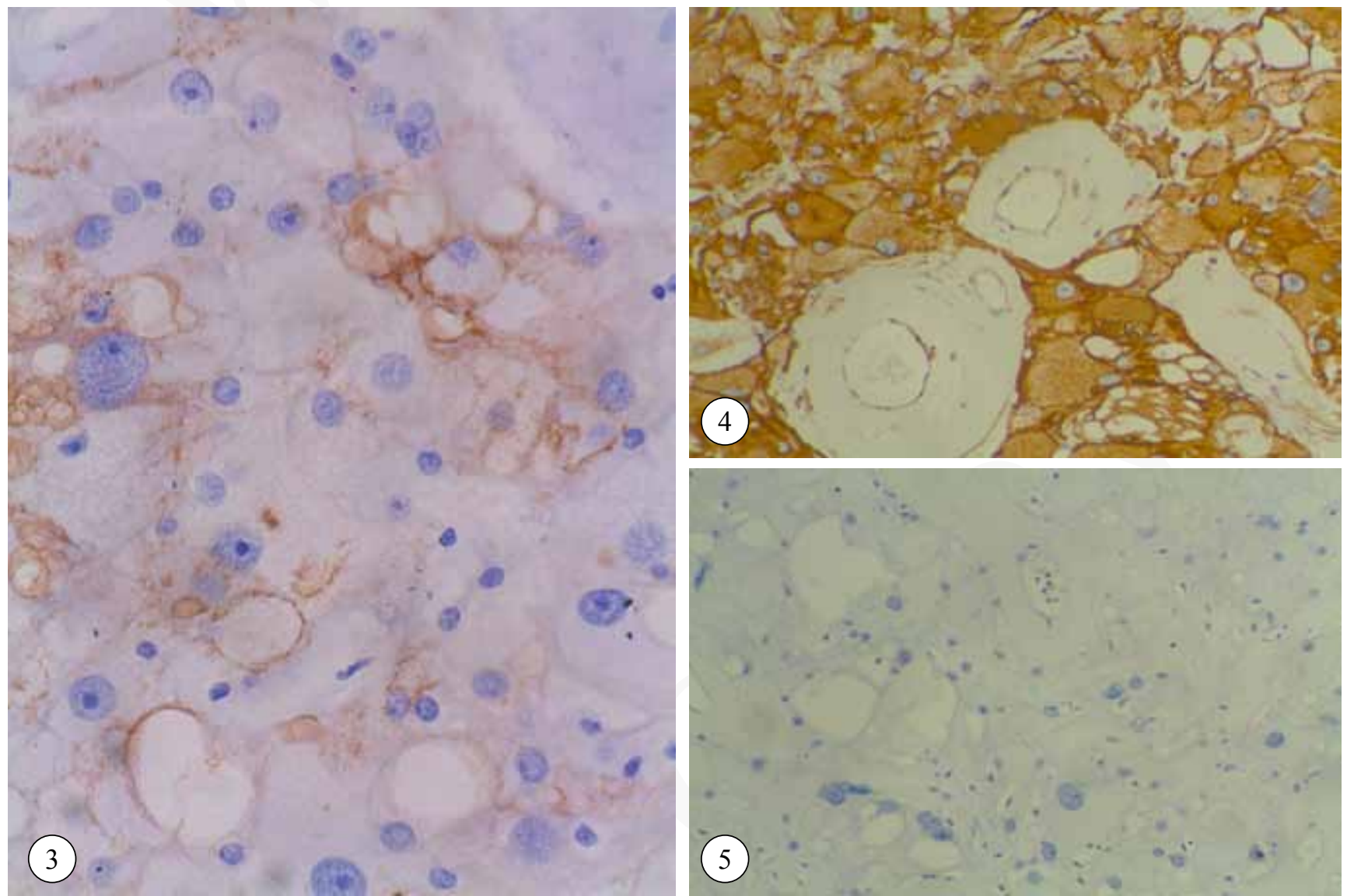

Figure 3: Epithelial Membrane Antigen showing cytoplasmic positivity (X50)

Figure 4: Vimentin showing diffue cytoplasmic positivity (X20)

Figure 5: Glial fibrillary Acidic Protein - Negative (X20)

Histological examination revealed tumor cells showing pleomorphic mononuclear cells with vesicular nucleus and few of the cells showing prominent nucleoli. The cells (fig.2A \& 2B) showed xanthomatous cytoplasm. Many hyaline vascular changes and cystic spaces were also noted. Small areas showed more recognizable meningothelial nests. The tumor cells were immunopositive for Epithelial Membrane Antigen (EMA, fig.3) and Vimentin (fig.4) and 
negative for Glial Fibrillary Acidic Protein (GFAP, fig.5). The diagnosis of microcystic meningioma was favoured.

\section{DISCUSSION}

Meningiomas are common benign tumours accounting for $13 \%$ to $26 \%$ of intracranial neoplasms. ${ }^{1}$ They are generally known to be solid tumors and their classical appearance on the CT scan and MRI usually lead to a correct diagnosis. ${ }^{4}$ Cystic meningiomas are rare subtypes constituting $4-7 \%$ of all meningiomas. ${ }^{5,6}$ Although this feature is unusual as meningiomas, this type of tumor has been described as one of the histological variants of meningiomas under the term of microcystic, myxomatous, vacuolated or arachnoid trabecular cell meningioma by various authors. ${ }^{2}$ Cystic meningiomas are more common in the pediatric age group than in adult. They are seen in $10 \%$ to $9 \%$ of all pediatric meningiomas, compared with only $2 \%$ to $4 \%$ in adult. ${ }^{2}$ Like other meningiomas the most frequent location of cystic meningiomas is on the cerebral convexity followed by biparasagital region with slight female preponderance, similar to most other meningiomas. ${ }^{2,7}$ The occurrence of a cystic tumor in a typical location for a meningioma in our patient with broad surface of contact with dura was suggestive of meningioma radiologically. Histologically Fukuoka et $\mathrm{al}^{8}$ believed that the cystic spaces within the cell clusters or sheets are characteristic of microcystic meningioma which was compatible with our microscopic findings. Microcystic meningioma is a distinct morphological variant of meningioma, characterized by loose texture and microcysts with formation of large extracellular spaces containing edematous fluid. ${ }^{9}$ The tumor cells have stellate and vacuolated cytoplasm with long cytoplasmic process. Meningiomas are generally considered to be mesenchymal origin, arising from the arachnoid membrane, although the tumors show distinct epithelial properties of interdigitating cytoplasmic processes and desmosomes. Their dual mesenchymal and epithelial properties are reflected by staining for both vimentin and EMA. ${ }^{2}$ Concerning the pathogenesis of the marked extracellular dilatation in microcystic meningioma, several theories have been proposed for the microcyst formation associated with secretory activity by the tumor cells, degenerative process, penetration of cerebrospinal fluid into tumor and vascular charges. ${ }^{9}$ Trabecular arachnoid cells found in tumor suggested that microcyst formation was an attempt by the neoplastic cells to recapitulate the subarachnoid space. ${ }^{10}$

The other differential diagnosis in a case of brain tumor with histolological findings showing xanthomatous cytoplasm ,hyalinized vascular changes and pleomorphic nuclei include pleomorphic xanthoastrocytoma, haemangioblastoma, angiomatous meningioma and microcystic meningioma. The immunohistochemical tests in our case revealed negativity for GFAP, diffuse positivity for vimentin and focal cytoplasmic positivity for EMA with the help of which the diagnosis of microcstic meningioma was made. Histologically pleomorphic mononuclear cells, xanthomatous cytoplasm, hyalinized vascular changes and microcystic spaces in the background in this case posed a diagnostic problem. Microcystic meningioma may occur in various age groups in contrast to pleomorphic xanthoastrocytoma which is predominantly seen in children without a significant gender bias. The tumor is located mostly supratentorial and $98 \%$ seen in temporal lobe. The other histological findings of pleomorphic xanthoastrocytoma which help to distinguish from microcystic meningioma include giant multinucleated neoplastic astrocytes, perivascular and intratumoral collection of small lymphocytes, eosinophillic granular bodies,negligible mitotic rate,eosinophillic hyaline droplets, Rosenthal fibres without necrosis and vascular proliferation. Reticulin stain shows dense intercellular reticulin network. ${ }^{11}$

\section{CONCLUSION}

Microcystic meningiomas are an unusual, but distinct morphological variant of meningioma, which are clinically and immunohistochemically similar to conventional meningiomas. Microcystic meningiomas showing cystic spaces, xanthomatous change in the cytoplasm of the tumor cells, pleomorphic cells is difficult to differentiate from other possible differential diagnosis. Immunohistochemistry has great role in differentiating microcystic meningioma from pleomorphic xanthroastrocytoma.

\section{REFERENCES}

1. Kleihues $\mathrm{P}, \mathrm{KC}$ Webster editors. World Health Organization Classification of Tumours of the Central Nervous System. 3rd edition. Lyon. International Agency for Research on Cancer(IARC) 2000.

2. Cho JH. Microcystic Meningioma-Unusual Variant Of Meningiomas. A case report . J. Korean Neurosurg Soc 2005;34:382-85.

3. Henry JM, Schwartz FT, Sartawi MA, Fox JL. Cystic meningiomas simulating astrocytomas. Report of three cases. J Neurosurg 1974;40:647-50

4. Quest DO. Meningiomas. An update. Neurosurgery 1978; 3:219-25

5. Odake G. Cystic meningioma: report of three patients. Neurosurgery 1992; 30:935-40.

6. Rengachary S, Batnitzky S, Kepes JJ, Morantz RA, O'Boynick P, Watanabe I . Cystic lesions associated with intracranial meningiomas. Neurosurgery 1979;4:107-14.

7. $\mathrm{Ng} \mathrm{HK}$, Tse CC, Lo ST :Microcystic meningiomas--an unusual morphological variant of meningiomas. Histopathology 1989;14:1-9

8. Fukuoka K, Hirokawa M, Kanahara T, Ishii N, Ishii R, Shirabe T : Cytology of microcystic meningioma in crush preparation. Diagn Cytopathol 2000;23:275-8.

9. Paik SS, Jang SJ, Park YW, Hong EK, Park HY, Lee JD :Microcystic meningioma-a case report-. J Korean Med Sci 1996;11:540-3.

10. Lantos PL, Vandenberg SR, Paul Kleihues. Tumors of the nervous systems.In :Graham. Lantos editors. Greenfields, Neuropathology. Arnold : 1997 . 732pp.

11. Burger PC, Scheithauer BW. AFIP Atlas of tumor pathology series 4 .Tumors of the central nervous system. Tumors of neuroglia and choroid plexus. American Registry of Pathology.4th series .Fascicles 7.pp107. 